\title{
Frequent Attenders
}

\author{
Andrew Crundell
}

\section{INTRODUCTION}

This report will provide an interim evaluation into the quality and outcomes of the Frequent Attender Team (FAT) based in the Royal Lancaster Infirmary. The report will provide some background to the service. The evaluation will explore aspects of the service structure, delivery and outcomes. This will allow for some conclusions to be made about the quality and success of the service and will result in some recommendations of how to improve the service and also improve the evaluation at 12 months to enable robust conclusions about quality and value for money to be made.

The evaluation provides a brief background and the drivers for the business case. There is a narrative detailing action undertaken during implementation and quantitative data illustrating outcomes. Outcomes are explored to understand whether patients' behaviour has changed or healthcare utilisation has been affected.

\section{FREQUENT ATTENDERS IN EMERGENCY DEPARTMENTS (20I4)}

\section{Summary of Recommendations}

I. Patients identified as 'frequent attenders' should be subject to senior decision maker review on each attendance to the Emergency Department

2. Patients who attend frequently should have a bespoke management plan to inform clinical management and enhance or standardise safe clinical care. This should be considered in all patients where such a plan will enhance clinical care (for example reducing unnecessary tests or provide clear analgesia strategy)

3. Patients identified as 'very high frequency attenders' (e.g. 30 or more attendances per year) should have a multidisciplinary meeting and case management; including social care and primary care, with a review of the bespoke management plan

4. Patients who are both 'frequent attenders' and exhibit challenging behaviours should be managed according to current guidance. This involves establishing and addressing underlying causes, whilst ensuring safety of patients and staff

5. Patients should be involved in all case management and in the production of care plans where possible

6. Persistent and recalcitrant challenging behaviours should only be subject to civil orders in exceptional circumstances

7. There should be a process of identifying 'frequent attenders' in all Emergency Departments, in order to enable implementation of the above. One commonly used method is to identify the current highest frequency attenders to a department

\section{Background \& Business Case}

In 20 |3/20 I 4, there were 604 emergency department attendances for 'Frequent Attenders' with an attendance level of 20 or more attendances in a 12 month period; this is estimated to cost $£ 73,000$ excluding emergency ambulance conveyance, inpatient spells and primary care costs.

A business case was developed to fund a Frequent Attender Scheme at the Royal Lancaster Infirmary. The service specification and KPIs were agreed in June 20I4. The investment was to fund I WTE band 6 specialist nurse and a 0.8 WTE band 3 administrator for a 4 month period; this was later translated into a 0.8 WTE band 6 specialist nurse and a 0.6 WTE band 3 administrator for a 6 month pilot period. This was later extended to $31 / 03 / 2016$.

\section{The Key Performance Indicators \& Expected Outcomes}

Monthly reduction of attendances of individual frequent attenders

Frequent attenders' experience of service

Reduction in attendances in ED

Reduction in re admissions

Improved user understanding of how to access appropriate support

\section{Implementation of Service}

Recruitment to the service was delayed. One nurse and an administrator were recruited in December 20 I4. Due to the nature of the service specification, time was allowed during implementation for some organisational development, such as awareness raising and training, and for some pathway and policy development. The service implemented a phased approach to developing the case load and became fully operational in April 2015.

The service was commissioned to solely operate on the Lancaster site; line management is from an Advanced Practitioner in the In-patient Pain Service and clinical leadership is provided by a Consultant in Emergency Medicine and/or the Speciality Team best placed to lead on an individual case.

The initial caseload of the 'Top 20' was identified through a Lorenzo report produced by one of the trust's Business Intelligence Analysts.

\begin{tabular}{|l|c|}
\hline North LANCS Patients & 18 \\
\hline Cumbria Patients & 2 \\
\hline ED Attendances & 517 \\
\hline Emergency Ambulances & 386 \\
\hline Inpatient Spells & 146 \\
\hline Total Bed Days & 236 \\
\hline $\begin{array}{l}\text { Collectively history of frequently attending; } \\
\text { ranging between 2-9 years }\end{array}$ & $\begin{array}{c}76 \text { Years } \\
\text { (Average 3.8 } \\
\text { Years) }\end{array}$ \\
\hline Total Cost to Commissioners & \\
\hline
\end{tabular}

The Service was designed based upon two best practice guidelines written by the College of Emergency Medicine in August 2014 ; Frequent Attenders in Emergency Departments 
and Care of Frequent Attenders at multiple Emergency Departments (FAMED) - this produced the 'I 0 steps' interventions to managing this group of patients and a Tier System based on suggested triggers, giving a description of likely attendance patterns and suggested actions/standard principles.

During the implementation stage the team also developed Standard Operating Procedures; including Lorenzo Alerts, Standard Patient Sign Posting Letters and GP Service Letters. This time also included discussions with hospital teams, community teams and other agencies expected to be closely linked with the service to provide congruent care throught out the healthcare community and wider in some cases.

\section{Primary Stakeholders involved in the Service included;}

Acute In-patient Pain Service

Adult Mental Health Liaison

Adult Social Care

Community Care Co-ordinators

Community Matrons \& Long-term Conditions Teams

Community Mental Health Care Co-ordinators

Court Mental Health Liaison

Emergency Department

GP Practices

Hospital Alcohol Liaison Service (HALS)

Housing Agencies \& Local Council

Independent Domestic \& Sexual Violence Advocate (IDSVA)

Lancashire Police - Early Action Team (EAT)

Lancashire Police - Neighbourhood Policing Teams

Local Security Management Specialist

Medical \& Surgical Admission Teams

NWAS Frequent CallerTeam

Paediatric Liaison

Safeguarding Adults \& Children

During the initial case reviews the primary presenting complaint was identified; this review was based on information available on Lorenzo and based on discussions with Emergency department staff regarding their understanding of the underlying issues that contribute to attendance, pre interventions.

\section{Primary presenting complaints:}

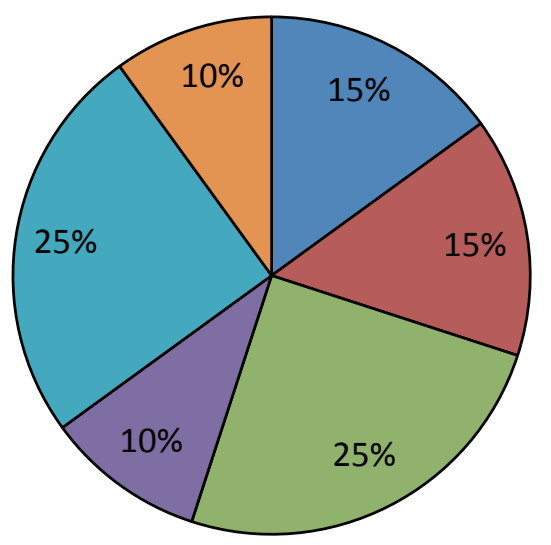

$\square$ Acute (UHMB)

$\square$ Alcohol (HALS)

$\square$ Behavioural

Children \& Young People

$\square$ Mental Health (LCFT)

$\square$ Substance Misuse

This information would also guide the primary interventions applied to all patients on the 'go live' date of April 2015 these included; Alerts, Signposting Letters and GP Letters.

\section{Primary Interventions}

Alerts; at the start of the project there was no clear way of identifying a frequent attender, the Alert process within Lorenzo was fostered - this alert contained no clinical information, but sought to identify the patient attendance pattern, promote the primary recommendation from the best practice guidelines that patients should be subject to senior review, this principle was also reinforced with a 'pop up' box so that every time a patient is selected this would be highlighted again without the need to open the alert; as this remained dependent on the staff member accessing this. Alerts also guided staff to relevant clinical notes and/or documents potentially relevant to the decision making process.

Patient Signposting Letters ratified by the Divisional Governance \& Assurance Group (Surgical) provided patients with an introduction of the service and what they might expect this to mean for their care, this also highlighted the attendance levels over the last 12 months and provided Choose Well Campaign information, providing both patient education and seeking to improve user understanding of how to access appropriate support. The key message is the use of A\&E Departments and 999 are for those who are seriously ill or emergencies. Patients attending over 20 times in 12 months are also assigned a case manager identified in the letter.

\section{Relevant signposting leaflets were also included;}

| | |

Bay Urgent Care

Choose Well

GP Extended Hours Service (Opening Doors Pilot)

Inspire (Alcohol \& Substance Misuse)

Lancashire Wellbeing Service (replacing Help Direct)

Late Night Pharmacy

Same Day Health Centre (Morecambe Area Patients)

Wellbeing and Mental Health Helpline (LCFT)

GP Letters, also ratified by the Divisional Governance \& Assurance Group (Surgical), provided patient GPs with an introduction to the service, highlighted the patient's attendance levels over the last 12 months; requesting a professional opinion on the issues faced, and offering the opportunity to contribute to any potential care plan that might be required in the Emergency Department. Responses are uploaded to Lorenzo and made available to decision makers; with the exception of GP Summaries as they are already available (up to date - live) via Lorenzo to the ED, Acute Medical Unit and Acute Surgical Unit.

\section{Secondary Interventions}

Patient Assessments take place in the Emergency Department or as an in-patient when admitted, although due to the increased out of hours and weekend presentations this did not allow for timely assessment of all patients, therefore delaying the development of a therapeutic relationship and understanding of patients' psychosocial element to their attendance(s): therefore, an outpatients clinic has been set-up to give the patients and team the opportunity to meet, review the case, attendances and care plan where in place. Patient engagement and attendance remains an issue as highlighted in the guidance. 
Multi-disciplinary Team (MDT) and/or Multi-agency Group (MAG) reviews took place in two forms; where patients needed a more detailed case review, patient specific MDT was required to understand and agree a detailed care plan, individual case conferences were organised and relevant stakeholders were invited always including the ED and GP, some case conferences were led by lead care teams (e.g. Mental Health Services or Lancashire Constabulary Early Action Team). Nine complex case conferences have been held to date.

Bi-monthly Multi-agency meetings continue to review the caseload, each agency's progress and consider which patients may benefit from a complex case conference.

The multi-agency quorum includes representatives from;

Community Care Co-ordinators

Emergency Department

HALS

Lancashire Care (Mental Health)

Lancashire Police

NWAS

UHMB Safeguarding

UHMB Security

To facilitate better working relationships and promote further multi-agency working, the team also linked in with a number of groups with Frequent Attenders appearing on most of these groups' agendas, these groups include;

\author{
Children \& Young People in the Emergency Department \\ Emergency Department Senior Team Meeting \\ GP Core Team Meetings \\ HALS Steering Group \\ Lancaster District Community Safety Partnership \\ Executive Group \\ Multi-Agency Oversite Group on Mental Health - North \\ LANCS \\ NWAS Frequent Callers Team \\ Royal Preston Hospital Frequent Attender MDT \\ Unscheduled Care Partnership - Mental Health \\ Violent Marker Group (UHMB Security)
}

A clinical note has been developed to allow for Care

Management Plans to be available on Lorenzo, or where care management plans have been developed by partner agencies these are scanned on to the system - locations of these documents are included in the Lorenzo Alert to guide Decision Makers to them during an episode of care. Some care plans are developed through MDT, and many seek to provide a detailed history and understanding of underlying issues; in some cases care management plans are prescriptive, and/or provide information whilst some are more security driven to manage challenging and anti-social behaviour.

\section{Care Plans are structured to include;}

Number of Previous ED Attendances

Primary Presenting Complaint(s)

Consent to Sharing of Care Plan

Active Alerts

Attendance History

Risk Assessment(s)

Medical History

Social History
ED Management Plan

Pain Management Plan

Admission Plan

Discharge Plan

Follow-up Plan

Agreed Plan Summary

Review Date (Maximum of 12 Months)

\section{Ongoing Interventions (Case Co-ordination)}

Facilitated Follow-up is an important element of case coordination; this group of patients has high levels of not attending planned outpatient follow-up, both following an inpatient spell and GP referral, this includes appointments at the hospital and/or other agencies; the team have come to understand why individuals do not attend appointments and reinforce the message of engagement and attendance at appointments to better manage their health, to support this the team use an SMS text reminder and organise patient transport where needed. In some, case care management plans highlighted the pattern of not attending planned follow-up and asked clinicians to consider this not be routine unless considered vital, and include this decision in Discharge Summaries for the patient's GP to refer back to the hospital if required.

Ongoing Review continued; when in hours face-to-face, or in the outpatient review clinic or by telephone follow-up, again due to the increased out of hours and weekend presentations. As therapeutic relationships developed, along with better understanding of the underlying issues and access to better information the team were able to provide more patient centred care and advice to patients, staff, carers, relatives and partner agencies.

Information Sharing posed a challenge initially, partly due to the infancy of the scheme, and a lack of clarity on how information would be used but also information governance, data sharing agreements and patient consent have been an ongoing topic for discussion throughout the pilot. Guidance clearly highlights the need for great information sharing especially with partner agencies and other emergency departments in the cases of FAMED patients.

\section{Vulnerable Adults, Children \& Safeguarding Concerns}

One of the clear suggestions of guidance supported by the findings of the team is the vulnerability of this patient group. Strong links have been formed with the UHMB Safeguarding Team to ensure that these concerns are identified, have/are being acted upon or have the appropriate safeguards in place and these concerns are highlighted for consideration at every presentation to the Emergency Department. A number of safeguarding concerns have been raised and through better understanding of safeguarding issues this has contributed to a better understanding of underlying issues that contribute toward attendance levels. Safeguarding and vulnerability of these patients is also a primary objective of staff training delivered to ED Staff. Safeguarding concerns in this patient group include;

\footnotetext{
Alcohol \& Substance Misuse (Including Opiate Seeking

Behaviours)

Children Looked After

Capacity \& Consent Issues (Deprivation of Liberties

Safeguarding)

Dangerous Offenders
} 
Domestic Violence

Fabricated and Induced IIIness (FII)

Healthcare Seeking \& IIIness Behaviour(s)

Mentally Disordered Offenders

Patients with Parental Responsibility

Personality Disorders

Self-Neglect

Sex Offenders

\section{Challenging \& Antisocial Behaviour}

Another suggestion of the guidance is an associated element of challenging behaviour in some cases, this again is supported by the team's findings; a close working relationship has been developed with both the UHMB Local Security Management Specialist, who links into the Area Security Management Specialist (especially in the management of FAMED Patients) and Lancashire Police to ensure that these concerns are identified, have/are being acted upon or have the appropriate safeguards in place and these risks are highlighted at every presentation to the Emergency Department, with Security involvement and/or security driven care management plans to ensure staff and patient safety. Clinical Incident submission has been encouraged to ensure management of such behaviours is in-line with trust policies and procedures, markers are in place. Behavioural concerns include;

Anti-social Behaviours (including Anti-social Behaviour Orders)

Absconding (Risk to Self)

Criminal Damage (Risk to Property)

Dangerous and Sex Offenders (Risk to Staff and Others)

Fluctuating Capacity (Clinical Factors)

Physical Abuse (Risk to Staff and Others)

Unreasonable Complainant Behaviour (Patient Advice and Liaison Service)

Verbal Abuse (Risk to Staff)

Zero Tolerance GP Scheme*

* The Zero Tolerance GP is presently not provided in North LANCS

\section{Patient Safety}

Patient Safety concern related to this group of patients often present decision makers with clinical and ethical dilemmas, this may be due to the lack of verifiable history available in Emergency Departments; especially when considering those patients seeking healthcare or frequently attending multiple emergency departments. Highlighting attendance levels, ensuring senior review and/or providing care management plans seeks to improve patient safety by providing strategies to;

Avoid Unnecessary and potential Harmful Investigations Ensure Safe Prescribing (including an Analgesia Strategy) Highlight any risk to Patient, Staff and/or Others

Provide Attendance and Verifiable History (especially from partner agencies)

Reduced Clinical and Ethical Dilemmas for Decision Makers

Support Safe Discharge and avoid Inappropriate Admission

Multi-agency working and ongoing monitoring also seek to ensure patient safety especially in cases with co-morbidities, as reduction in attendance is seen the team engage with patients and primary care providers to ensure the health and wellbeing of patients is not compromised by promoting that
A\&E Departments and 999 Services are for those who are Seriously III or Emergencies; supported by patient education in how to better access appropriate support.

\section{Specialised Training}

Specialised Training has been delivered to Emergency Department, training takes place face-to-face, the following learning objectives are covered;

Understand the Characteristics of Frequent Attenders to Emergency Departments

Understand the Characteristics of FAMED Patients

Understand the Risks Associated with Frequent Attenders

Understand the Basic Principles of Managing Frequent Attenders

Understand how the UHMB plans to Manage Frequent Attenders

59. I\% of RLI Emergency Department staff has been trained promoting two key principles of the pilot;

Should be reviewed by Senior Clinical Decision Maker Manage Vulnerable Patients, Consider Safeguarding Issues in Every Case

\section{RESULTS}

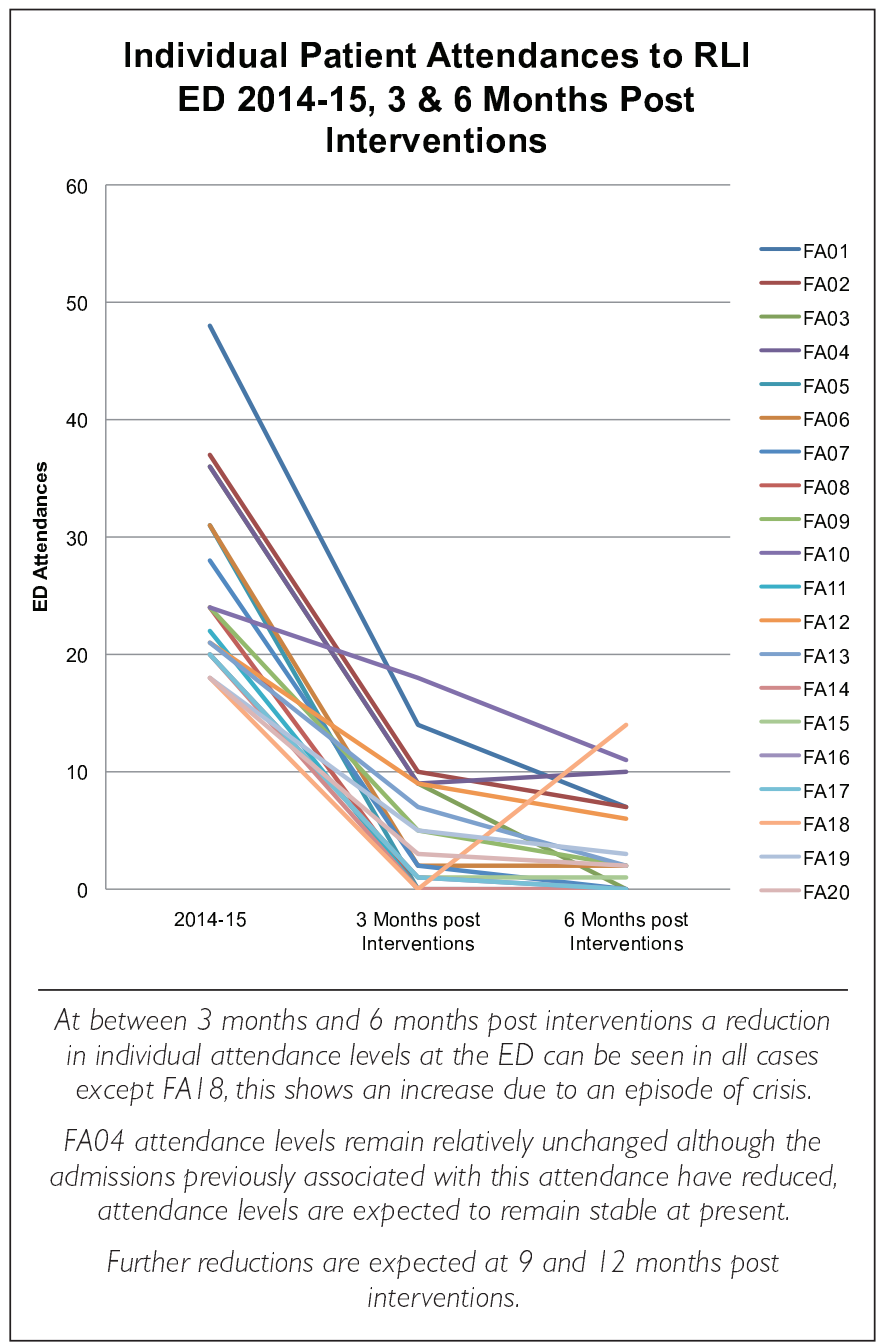




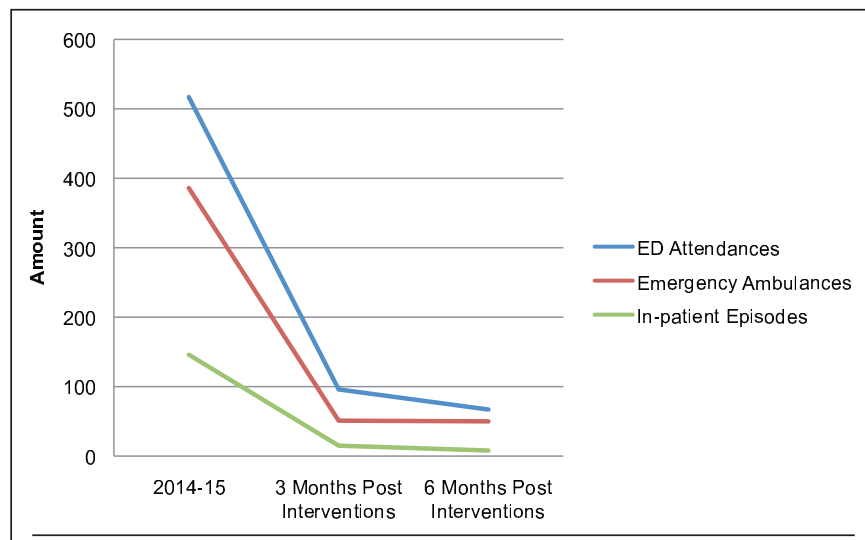

At 3 months post initial interventions ED attendances and Admissions begin to reduce, ambulance remains constant.

Further reductions are expected at 9 and 12 months post interventions.

\section{Cost savings for Commissioners (6 Months)}

\begin{tabular}{|l|c|c|c|}
\cline { 2 - 4 } \multicolumn{1}{c|}{} & $2014-$ I5 & $\begin{array}{c}\text { April I5 } \\
\text { - July I5 }\end{array}$ & Reduction \\
\hline ED Attendances & 517 & 164 & $36 \%$ \\
\hline $\begin{array}{l}\text { Emergency } \\
\text { Ambulances }\end{array}$ & 386 & 101 & $48 \%$ \\
\hline Inpatient Spells & 146 & 23 & $68 \%$ \\
\hline Total Bed Days & 236 & 23 & $81 \%$ \\
\hline $\begin{array}{l}\text { Total Cost to } \\
\text { Commissioners }\end{array}$ & & & \multicolumn{1}{c}{} \\
\hline
\end{tabular}

Total Saving

\section{Increased Caseload and Progression}

Following the extension of the project the team lowered the threshold for attendance to be included in the project to 12 attendances in a 12 month period, this established an initial caseload of 48 patients, with a further 31 patients identified to date. Of these 79 cases 28 no longer attend above the threshold, effectively closing these cases; although alerts remain in place until the end of the pilot to ensure episodes of crisis can be responded to.

Similar reductions in attendance and admission can be seen in the majority of cases, although some complex cases require sustained ongoing intervention, and multi-agency working to manage - possibly due to the learnt behaviours associated with health seeking. The new threshold allows for earlier identification of potential underlying issues to accessing unscheduled \& emergency care, with this system for identification in place the team are better equipped to be proactive as opposed to re-active as has been the case due to the nature of the scheme and its infancy.

\section{Further Development}

The Frequent Attender Team feel that most patients within their caseload required intensive, sustained support over longer periods of time to address their entrenched multiple and complex needs to achieve the desired end; through patient - professional negotiation, intensive engagement and congruency and therefore feel the pilot should continue for a further 12 months to further address the complex needs of those patients where sustained healthcare seeking and/ or inappropriate access to emergency services has led to the need to facilitate change of health behaviour.

A similar caseload is reflected on the Furness General site, with some Cumbria CCG patients attending the RLI site. The Team feels in the second year of the project the FGH patients should be included in the scheme.

\section{Step Principle}

University Hospitals NHS of Morecambe Bay NHS Foundation Trust

\begin{tabular}{|l|l|r|r|}
\hline & In-hospital Team Action & Intervention & \multicolumn{1}{|c|}{ Aim } \\
\hline 1 & Case Study/Review & Alert & Ensure Senior Review in the ED \\
\hline 2 & Sign Posting Letter \& Information & Patient Letter & Sign Posts Patients to other Services \\
\hline 3 & MDT Opinions & GP Letter & Ensures GP Aware-Management Plan \\
\hline 4 & Case Manager Assignment & Patient Letter & Provides Specialist Management \\
\hline 5 & Patient Assessment & Face-to-Face & Assessment including Psychosocial \\
\hline 6 & Bespoke Management Plan & Informal Plan & Informal Plan Specialist Nurse/Patient \\
\hline 7 & Facilitated Follow-Up & Telephone & Ensures Follow-Up OPA Attended \\
\hline 8 & MDT Case Conference & Formal Plan & Multi-Disciplinary Assessment \\
\hline 9 & MDT Risk Assessments & Formal RA & Multi-Disciplinary Risk Assessment \\
\hline 10 & Multi-Agency Information Sharing & Audit & Consistent Patient Care \\
\hline & & & \\
\hline
\end{tabular}




\section{University Hospitals NHS of Morecambe Bay \\ NHS Foundation Trust}

\section{OChoose wrwwhs.uk}

Frequent Attenders Team

Royal Lancaster Infirmary

Ashton Road

Lancaster

LA1 4PR

Tel: 01524511975

e-mail: frequent.attenders@mbht.nhs.uk

Web: www.uhmb.nhs.uk

Date

PRIVATE \& CONFIDENTIAL

Dear

\section{RE: Attendance Levels at the Royal Lancaster Infirmary}

We are writing to you regarding our concern around your frequent attendance at hospital.

We notice you have attended ? times in the last 12 months.

As a result of this, I write to you regarding being reviewed by the Frequent Attender Team. This is a new service offered by Lancashire North CCG based in University Hospitals of Morecambe Bay at Royal Lancaster Infirmary.

\section{What does this mean to me?}

We will be working closely with the Emergency Department (A\&E), Hospital Specialists and Specialist Teams, the Ambulance Service and your GP to look at the ways we can all work together to reduce the number of times you need to attend, endeavour to deliver the best care possible and put plans in place to manage your health and wellbeing.

The aim of this service is to provide the safest and most effective care the Trust can provide in a realistic time frame and in the most suitable location.

In some more complex cases you may be assigned a Case Manager from the Team especially if you find yourself admitted to a ward frequently.

\section{Please Remember A\&E Departments and 999 Services are for those who are Seriously III or Emergencies}

Read on to find out more about which service is most suitable for your illness or injury;

Westmorland General Hospital

Burton Road

Kendal

LA9 7RG

CHAIR: PEARSE BUTLER

Tel: 01539732288

CHIEF EXECUTIVE: JACKIE DANIEL 


\section{Self-Care}

Self-care is the best choice to treat very minor illnesses and injuries. A range of common illnesses and injuries can be treated at home simply by combining a well-stocked medicine cabinet with plenty of rest.

NHS 111

NHS 111 offers confidential health advice and information by telephone, 24 hours a day. Contact NHS 111 to check your symptoms and find health services in your local area.

Choosing well ensures you get expert advice in the shortest possible time, meaning busy NHS services can help those who need them most.

\section{Pharmacists}

Pharmacists can be found across Lancaster, Morecambe, Carnforth and Garstang. Visit your pharmacy when you are suffering from health problems that do not require being seen by a nurse or doctor.

Your pharmacist can provide advice regarding illnesses and suggest the best medicines to treat them.

To find your local pharmacy visit www. nhs.uk and click on 'Find and Choose Services' or phone NHS Direct on 08454647.

\section{GPs}

GP surgeries can be found across Lancaster and Morecambe. If you have an illness or injury that just will not go away, make an appointment with your local GP. They provide a range of services by appointment, including medical advice, examinations and prescriptions. When absolutely essential, GPs can also provide home visits out of hours.

To find GP surgeries near you visit NHS Choices www.nhs.uk or phone NHS Direct on 08454647.

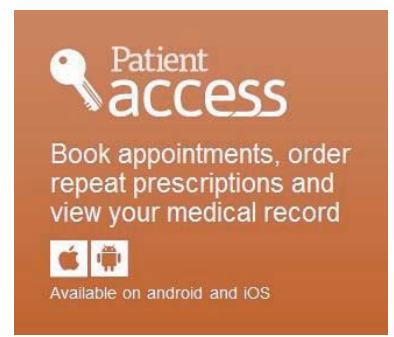

Walk in centers

Walk-in centres, minor injuries units and same day centres offer convenient access to a range of treatment for minor illnesses and injuries including infections, rashes, fractures and lacerations, emergency contraception and advice, stomach upsets, cuts, bruises, burns and strains.

\section{A\&E 999}

Accident and Emergency departments (A\&E) should only be used in a critical or lifethreatening situation. A\&E departments provide immediate emergency care for people who show the symptoms of serious illness or are badly injured. Dialing 999 and stating a medical emergency will result in a response vehicle being sent to your location.

Choosing well ensures you receive the rest and recovery you need, meaning busy NHS services can help those who need them most.

\section{Information Advice Service}

The Information Advice Service is a support and information service for all adults aged 18 and over across Lancashire. This service is designed to help people get the right practical support, information and advice that they need before a small problem becomes a crisis.

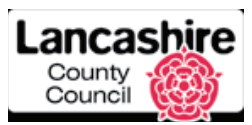

Tel: 03033331111

http://www.lancashire.gov.uk/

We have included some leaflets that we feel might also be helpful to you having looked at the reasons why you attend the Emergency Department.

If you would like any further information on this matter please do not hesitate to contact a member of our team. We will also be writing to your GP to introduce the service and ensure your case is reviewed by your GP.

The team is available to you should you need to speak to us about any element of your care, or require help in accessing community services in your area.

Again please do not hesitate to contact us with any further questions, or for any help we can offer.

Yours sincerely

Frequent Attenders Team

Royal Lancaster Infirmary 